\title{
Different Protocols of Physical Training: Effect on Markers of Oxidative Stress in Erythrocytes in Rats
}

\author{
Marcelo Costa-Junior ${ }^{1}$, Wener Barbosa-Resende1, Michel Barbosa de Araújo², \\ Rodrigo Augusto Dalia ${ }^{3}$, Leandro Pereira de Moura ${ }^{4}$, Luciana Alves de Medeiros' ${ }^{1}$, \\ Lucas Moreira Cunha ${ }^{1}$, Eliete Luciano ${ }^{3}$
}

${ }^{1}$ Federal University of Uberlandia, UFU, Uberlandia, Brazil

${ }^{2}$ University Catholic Center of Quixadá, Quixáda, Brazil

${ }^{3}$ Institute of Biosciences, UNESP, Rio Claro, Brazil

${ }^{4}$ School of Applied Sciences, Unicamp, Limeira, Brazil

Email: marcelotmcosta@hotmail.com

How to cite this paper: Costa-Junior, M., Barbosa-Resende, W., de Araújo, M.B., Dalia, R.A., de Moura, L.P., de Medeiros, L.A., Cunha, L.M. and Luciano, E. (2018) Different Protocols of Physical Training: Effect on Markers of Oxidative Stress in Erythrocytes in Rats. Open Access Library Journal, 5: e4411.

https://doi.org/10.4236/oalib.1104411

Received: February 6, 2018

Accepted: March 9, 2018

Published: March 12, 2018

Copyright (อ 2018 by authors and Open Access Library Inc.

This work is licensed under the Creative Commons Attribution International License (CC BY 4.0).

http://creativecommons.org/licenses/by/4.0/

(c) (7) Open Access

\begin{abstract}
The imbalance between oxidant molecules and antioxidant agents is characterized as oxidative stress (OS) and may lead to severe damage to the organism. In contrast, the physical training of aerobic and resistive character promotes increases of the antioxidant response, resulting in a balance and/or minimizing damage. Therefore, the objective of the study is to verify the effect of aerobic training, resistive training and concurrent training on oxidative stress of Wistar rats. Methods: To perform the experiment, 40 Wistar rats were subjected to eight weeks of training. The animals were distributed into 4 different groups: control, aerobic, resistive and concurrent (combination of aerobic and resistive protocols in the same section) an h/day, five days/week, for eight weeks. At the end of the experimental period: the antioxidant enzymes (catalase, glutathione peroxidase and superoxide dismutase), biomarkers of oxidative stress (TBARs and $\mathrm{H}_{2} \mathrm{O}_{2}$ ) as well as the activity of reduced and oxidized glutathione were analyzed. Results: Regarding the markers, TBARs and $\mathrm{H}_{2} \mathrm{O}_{2}$ trainings were not effective in promoting significant reduction. In contrast, the three training protocols caused an increase in SOD and GSH-GPx enzymes. Conclusion: Therefore, we concluded that both training protocols promoted benefit to the organism, because they caused the increase of antioxidant enzymes without disturbance in the pro-oxidant markers.
\end{abstract}

\section{Subject Areas}

Bioinformatics, Biophysics, Biotechnology, Zoology 


\section{Keywords}

Oxidative Stress, Exercise, Antioxidant Enzymes

\section{Introduction}

The finding that the physical exercise promotes acute increase in Reactive Oxygen Species (ROS) has been reported for over 30 years [1]. After this discovery, some evidences indicated that despite high concentrations of ROS induced damage to lipids, proteins and nucleic acids [2] [3], low to moderate levels of these oxidizing molecules played an important role in the signaling and regulation of several genes related to antioxidant capability and physical performance [4] [5] [6] [7].

According to Davies et al. [8] the increase in production of ROS during exercise occurs mainly in muscle fibers. From this assumption, several studies assume skeletal muscle as a key producer of ROS and blood as a general representation of the organism [8] [9] [10].

However, according to Nikolaidis et al. [11] erythrocytes are a major source of ROS production in the blood, especially hydrogen peroxide $\left(\mathrm{H}_{2} \mathrm{O}_{2}\right)$, both at rest and after an exercise session [12] [13] [14]. Moreover, the blood can interact with tissues and organs and, consequently, with possible producers sources of ROS, dramatically increasing levels of oxidant molecules in erythrocytes [11] [15].

Physical training is a condition that is capable of generating cellular adaptations, both in tolerance to oxidative changes as in the improvement of antioxidant system [6] [9] [16] [17].

The beneficial effects of aerobic exercise training on antioxidant defense mechanisms in several tissues have been reported [12] [18]. Revan and Erol [19] have observed in subjects who performed a protocol of physical training in the intensity of $50 \%$ to $70 \%$ of maximum heart rate. It was able to reduce the levels of lipid peroxidation in erythrocytes after exhaustive exercise. Corroborating these findings, Oztasan et al. [20] have found in rats the effect of aerobic exercise training on oxidative stress in erythrocytes. According to these authors, aerobic training was effective in preventing stress induced by acute exercise with consequent increased activity of antioxidant enzymes.

Furthermore, the effects of resistive training (RT) on the oxidative stress (OS) also have been studied in humans [21] [22] and animals [18] [23] [24].

Some authors have observed that the RT promoted adaptation in humans, showing lower levels of lipid peroxidation [22] and increased activity of superoxide dismutase and catalase enzyme in erythrocytes [21]. In animals, the existing studies verified the effect of the RT in skeletal muscle [23], cardiac [24] and liver [18]. Murlasits et al. [23] have reported that RT three times a week promotes a $66 \%$ increase in the expression of the enzyme superoxide dismutase 
(SOD). In contrast, Ahmadiasl et al. [24] found no improvement in the antioxidant capacity of rats subjected to jumping protocol after 4 and 12 weeks of training at rest. However, there was no increase in oxidative parameters after acute exercise, showing that this type of training ameliorates the deleterious effects of acute exercise in relation to oxidative stress.

Physical training, both aerobic and resistive, can result in adaptations in antioxidant capacity, thereby protecting body cells against the deleterious effects of ROS. Nevertheless, few studies have been conducted about the effect of combined physical exercise on oxidative stress and antioxidant enzymes of Wistar rats, making clear the need for studies comparing different protocols of physical training. Moreover, it is unclear whether different training programs have similar responses in relation to the oxidative markers, or the combination of trainings promotes an antioxidant adaptation more significant than the training alone. Thus, the objective of this study was to investigate the effect of different protocols of physical training on markers of oxidative stress in erythrocytes of rats.

\section{Materials and Methods}

\subsection{Animals}

Forty male Wistar rats with 90 days old (at the beginning of experiment) were selected from the Central Biotery Facility of the São Paulo State University (UNESP), Botucatu. The animals were housed in polyethylene cages, measuring $37 \times 31 \times 16$ (five rats per cage), under controlled temperature conditions $21^{\circ} \mathrm{C}$ and photoperiod (12 hours light/dark), fed with a standard rat chow (Purina) and water "ad libitum" for the experimental groups (in the Biotery of Biodynamic Laboratory of the Department of Physical Department, Institute of Bioscience, UNESP, Rio Claro). All experimental procedures were approved by the Ethics Committee on Animal Use (CEUA-6777).

\subsection{Training Protocols}

The animals were randomly distributed into four groups: sedentary (S), aerobic training (AT), resistive training (RT) and concurrent training (CT). All animals were adapted to the liquid medium before starting the training protocol. The training protocols were performed in a periodized way, as suggested by Araújo et al. (2012). After an adjustment period, the three trained groups (AT, RT and $\mathrm{CT}$ ) were subjected to a training program conducted five times a week for eight weeks. The groups AT and CT were subjected to Lactate Minimum Test before, after four and eight weeks of training. The groups CT and RT were subjected to the five repetition maximum strength test (5-RM) before, after four and eight weeks of training.

\subsection{Adaptation to the Liquid Medium}

The adaptation to the liquid medium was performed to all animals prior to their testing and training protocols. Adaptation took 10 days to complete and was 
done in the same tanks where the trainings occurred, with the water temperature kept at $31^{\circ} \mathrm{C} \pm 1{ }^{\circ} \mathrm{C}$. The goal of this process was to reduce the stress of the animals without promoting physiological adaptations resulting from physical training, and also to adapt them to the execution of the Lactate Minimum Test and to the proposed physical trainings. The rats were kept in the water at a $5 \mathrm{~cm}$ depth for five, ten and fifteen seconds during the three first days. From the fourth to the sixth day the animals were put to swim in a 70-cm-deep water tank for five, ten and fifteen seconds, respectively. On the following two days the animals had a load corresponding to $3 \%$ of their weight attached to their backs and were then submitted to a swimming routine for five minutes on the seventh day, and ten minutes on the eighth day. The last two days consisted of increasing the time of exercise to 15 minutes, with the further addiction of a 30 -second stimulus with a $10 \%$ of body weight load in order to keep the animals from panicking during the exercise in the hyperlactacidemia induction phase, which could possibly affect the test. Introducing the $3 \%$ and $10 \%$ of body weight loads aimed to soften the stress caused by the burden and promote a greater success percentage during the Lactate Minimum Test, which was performed 24 after the tenth adaptation day.

\subsection{Lactate Minimum Test}

The test was applied to determine the anaerobic threshold (AnT) [25] [26] of the animals using a modified procedure [27]. This test involves three steps: 1) high-intensity exercise to cause an increase in circulating blood lactate; 2) recovery to ensure high levels of lactacidemia and 3) exercise test with progressive loads, with blood sampling for lactate analysis at each load.

For the hyperlactacidemia induction, the animals were placed individually in tanks $(100 \times 80 \times 80 \mathrm{~cm})$, containing water at $31^{\circ} \mathrm{C} \pm 1^{\circ} \mathrm{C}$, supporting a load of $13 \%$ body weight and were subjected to swimming for 30 seconds. After 30 seconds of rest, they were subjected to swimming again with the same loads until exhaustion. After a rest period ( $9 \mathrm{~min})$, blood samples were collected by an incision in the tip tail and the animals began the swimming exercise with progressively higher intensities [27] [28]. The first load was equivalent to $4 \%$ body weight (bw) being increased by $0.5 \%$ bw every 5 minutes, until exhaustion of the animal. At each load change, blood sample was collected for lactate dosage [29]. The determination of blood lactate levels was performed by enzymatic method [30].

\subsection{Adaptation to Resistive Training}

The process of adaptation to the jump of the animals resistive and coucorrent training was started 48 hours after the Lactate Minimum Test, and lastedfive days. During all the procedure the adaptation jump of the animals the aerobic training was submitted to a load corresponding to $90 \%$ of the lactate minimum, with a progressive time increase until they had performed 60 minutes of train- 
ing.

The animals were initially put into shallow water, with a load of $50 \%$ of their body weight attached to their backs on a Velcro ${ }^{\oplus}$ bag. On the twelfth adaptation day, the water level was progressively increased to $150 \%$ of the naso-anal length so the animals could perform 10 jumps. On the two next days the rats performed two sets of 10 jumps with a load weighing $50 \%$ of their body weights and an interval of one minute and a half between sets. On the last adaptation day before the five maximum jump test, the animals executed two sets of 10 jumps with a load of $70 \%$ of their body weight so they could get close to the maximum load.

The jumps occurred in a $250 \mathrm{~mm}$ diameter PVC pipe aiming to limit the directions the animal could follow, thus favoring the jump. The holed-bottom pipe was put in $100-\mathrm{cm}$ length $70-\mathrm{cm}$ wide asbestos tanks, with water at a depth corresponding to $150 \%$ of the body length. Jump standardization was done following Rogatto et al. (2004).

\subsection{The Five Repetition Maximum Strength Test (5-RM)}

To evaluate the animals muscle strength, it was used the jump system in tanks with water [32]. However, we used the five repetition maximum strength test (5-RM) to determine the maximum load of animals. Moreover, 48 hours elapsed after the adaptation period already described, the animals of groups CT and RT started the 5-RM test, swimming for one minute without load. After the one-minute rest, the animals were subjected to two series of physical preparation, the first series of 10 jumps with load of $50 \%$ bw. In the second series of physical preparation, 5 jumps were conducted with a relative load of $70 \%$ bw. After five minutes of rest the animals initiated the 5-RM test, which was composed of a maximum of five attempts with recovery interval of five minutes between each attempt. The 5-RM test was validated when the animals in all jumps exceeded at least the snout of the established marking (150\% naso-anal length). If successfully completed, the test was increased a load to the dorsum of the animal, if the test was given as invalid, the load was removed from the dorsum of the animal. The final load found was considered $100 \%$ of 5-RM. The tests were always at similar time with a maximum of five attempts per day.

\section{Protocols of Physical Intervention}

\subsection{Protocol of Aerobic Training}

The animals of groups aerobic training were subjected to swimming exercise in individual tanks containing water at $31^{\circ} \mathrm{C} \pm 1^{\circ} \mathrm{C}$, one hour a day, five days a week, totaling eight consecutive weeks, supporting overload of lead tied to the thorax. Initially, the training load was equivalent to $90 \%$ of AnT individual, identified by the lactate minimum test, with an increase of $5 \%$ weekly until the third week, with intent to cause an overload and reduce the chance of the animal entering in a state of training monotony [28]. Throughout the experimental protocol the animals submitted to aerobic training maintained the exercise for 
60 minutes from Monday to Friday. In the fourth week, the animal maintained a load of $100 \%$ AnT, which promoted a reduction in volume in the last three days of training for $75 \%$ initial time (45 minutes) in order to promote a supercompensation [31] and, at the end of the week, the AnT test was carried out again to a new adjustment of the load. This training cycle was repeated until the eighth week. All training sessions were initiated in the afternoon (18:00).

\subsection{Protocol of Resistive Training}

The animals selected for resistive physical training conducted jumping exercises into water tanks, with a load attached in the thorax (backpack lead). Each exercise session consisted of eight series of 8 to 12 jumps, with an interval of one minute between each series. The training was carried out five days a week, with the weekends as breaks for a period of eight weeks in a row.

The jumps were performed on PVC tube, individually, $100 \mathrm{~cm}$ long, $70 \mathrm{~cm}$ diameter, with water at a depth corresponding to $150 \%$ of a rat body length, and the jump being counted as effective only when the animal succeed in overcoming this water height limit [32]. The periodization of training was made in order to avoid the training monotony, which according to Kuipers [33] and Araújo et al. [28] may lead to overtraining. The initial load was equivalent to $80 \%$ of $5-\mathrm{RM}$ predetermined, with an increase of $5 \%$ weekly, until the third week. In the fourth week, the animal kept a charge of $90 \%$ of $5-\mathrm{RM}$, with a reduction in volume in the last three days of training to $75 \%$ of the initial (6 series) aiming to promote supercompensation [31] and, at the end of that week, the 5-RM test was carried out again for a new adjustment of the load. This training cycle was repeated until the eighth week. All training sessions were initiated in the afternoon (18:00).

\subsection{Protocol of Concurrent Training}

The animals subjected to Concurrent Training protocol performed a combination of swimming exercise for 30 minutes and 4 series of jumping exercise, five times a week for 8 weeks, following the same periodization protocol of aerobic and resistive training.

\subsection{Obtaining Biological Material}

At the end of the experiment the animals were anesthetized with $\mathrm{CO}_{2}$, after resting for 48 hours to euthanasia. Blood was collected through cardiac puncture directly of the left ventricle and, immediately after, the blood was centrifuged three times at $2.500 \mathrm{rpm}$ for 5 minutes in $0.05 \mathrm{~N}$ phosphate buffer (composition, in $\mathrm{g} / \mathrm{L}: \mathrm{KH}_{2} \mathrm{PO}_{4}, 1.34$ and $\mathrm{NaHPO}_{4} \cdot 2 \mathrm{H}_{2} \mathrm{O}, 7.1$ ) with $\mathrm{NaCl} 0.9 \% \mathrm{pH}$ 7.4. After isolation of red blood cells, they were lysed by adding $\mathrm{H}_{2} \mathrm{O}(1: 1 \mathrm{v} / \mathrm{v})$ and centrifuged at $5.000 \mathrm{rpm}$ for 10 minutes twice, in order to remove the remaining membranes, and frozen at $-80^{\circ} \mathrm{C}$ for later analysis. The hemoglobin dosage of the hemolysate was made by the method of BEUTLER, (1975). 


\subsection{Biomarkers of Lipid Peroxidation: Concentration of Products That React to Thiobarbituric Acid (TBARs)}

The thiobarbituric acid reactive substances (TBARS) was measured to quantify the products of lipid peroxidation in erythrocytes. This method consists in the analysis of the final products of lipid peroxidation (lipid peroxides, malondialdehyde, and other low molecular weight aldehydes).The substances react with thiobarbituric acid to form colored species and its concentration can be determined spectrophotometrically at $532 \mathrm{~nm}$ [34].

\subsection{Biomarkers of Antioxidant Defense System}

\section{Enzymatic activity catalase (CAT)}

The assays for dosage of catalase activity were carried out by adding to the erythrocyte samples: $50 \mathrm{mM}$ phosphate buffer and hydrogen peroxide $\left(\mathrm{H}_{2} \mathrm{O}_{2}\right) 10$ $\mathrm{mM}$, (AEBI, 1984). The calculation of catalase activity was made by the following equation: $(2.3 / \Delta \mathrm{t}) .(\mathrm{a} / \mathrm{b}) .(\log \mathrm{A} 1 / \mathrm{A} 2)$, where: $\mathrm{a}=$ volume of sample in $\mathrm{cu}-$ vette; $\mathrm{b}=$ total volume of cuvette; $\mathrm{A} 1=$ absorbance value at $\mathrm{t}=0$ and $\mathrm{A} 2=\mathrm{ab}$ sorbance values of the final time, in our case, it happened at 15 seconds after the reaction initiation (AEBI, 1984).

Enzymatic activity superoxide dismutase (SOD) and Glutathione peroxidase (GSH-GPx)

The superoxide dismutase (SOD) and glutathione peroxidase (GSH-GPx) activity in erythrocytes was determined using SOD assay kit number 706002 and GPx assay kit number 703002 (Cayman Chemical Company, Ann Arbor, Michigan).

\section{Reactive oxygen species: Hydrogen Peroxide $\left(\mathrm{H}_{2} \mathrm{O}_{2}\right)$}

The hydrogen peroxide $\left(\mathrm{H}_{2} \mathrm{O}_{2}\right)$ in erythrocytes was measured using Amplex ${ }^{\oplus}$ Red Hydrogen Peroxide/Peroxidase Assay Kit number A22188 (Thermo Fisher Scientific, Molecular Probes ${ }^{\mathrm{TM}}$ ).

\section{GSH/GSSG}

The concentrations of reduced and oxidized glutathione (GSH and GSSG) were determined by fluorometry after precipitation of proteins with meta-phosphoric acid $25 \%$, in order to obtain the glutathione concentration in the erythrocytes. The analysis was performed according to the method described by Hissin and Hilf (1976) which utilized the reaction between GSH and ortho-phthaldehyde (OPT) $1 \mathrm{mg} / \mathrm{mL}$ in phosphates buffer $0.1 \mathrm{M}$-EDTA $0.005 \mathrm{M}$, $\mathrm{pH} 8.0$ and, between GSSG and OPT in NaHO $0.1 \mathrm{~N}, \mathrm{pH} 12.0$, so that the $\mathrm{N}$-ethylmaleimide (NEM) $0.04 \mathrm{M}$ might prevent the interference of GSH on measurements of GSSG. Emission spectra and excitation of GSH and GSSG showed the maximum excitation at $340 \mathrm{~nm}$ and emission at $430 \mathrm{~nm}$. The values are expressed in $\mu \mathrm{mol} / \mathrm{mg}$ of $\mathrm{Hb}$.

\section{Statistics}

Data normality was verified using the Shapiro Wilks test, homogeneity of variance was analyzed by Bartlett test. All experimental results were analyzed using 
the t-student test or by analysis of variance two-way (ANOVA), where appropriate. When necessary we used pos hoc bonferroni test. For all analyses, $p<$ 0.05 was considered significant.

\section{Results}

Mean values \pm standard error of body mass during the weeks of the protocol experiment is expressed in Figure 1(a). Figure 1(b) shows a significant difference of the TA and TR groups in relation to the GC in the area under the curve (BSA) of body mass development, that is, a body mass miracle during the experiment weeks.

Regarding the TBARs, no significant difference was found between groups, i.e., the physical training provided no reductions in the levels of lipid peroxidation (Figure 2).

As observed in Figure 3, the training did not modify the concentration of $\mathrm{H}_{2} \mathrm{O}_{2}$.

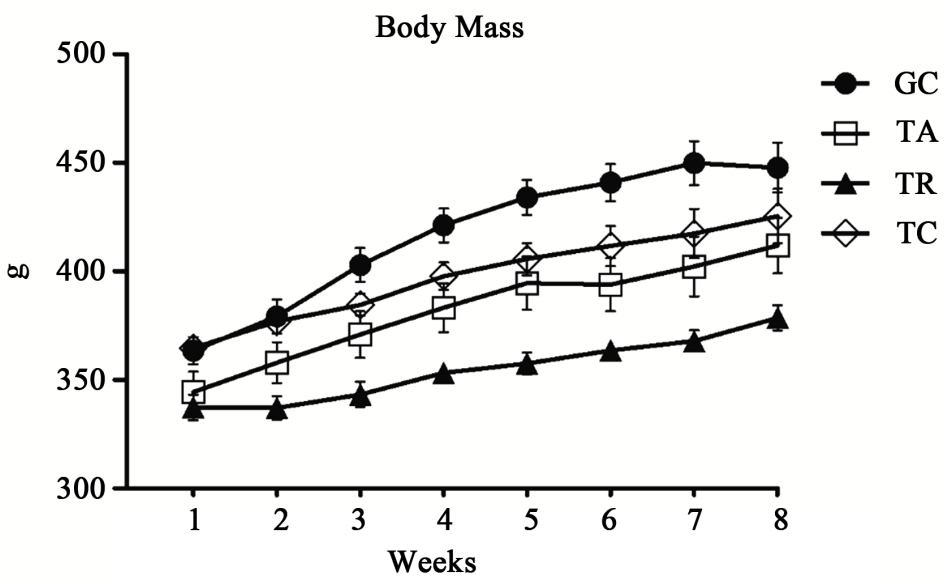

(a)

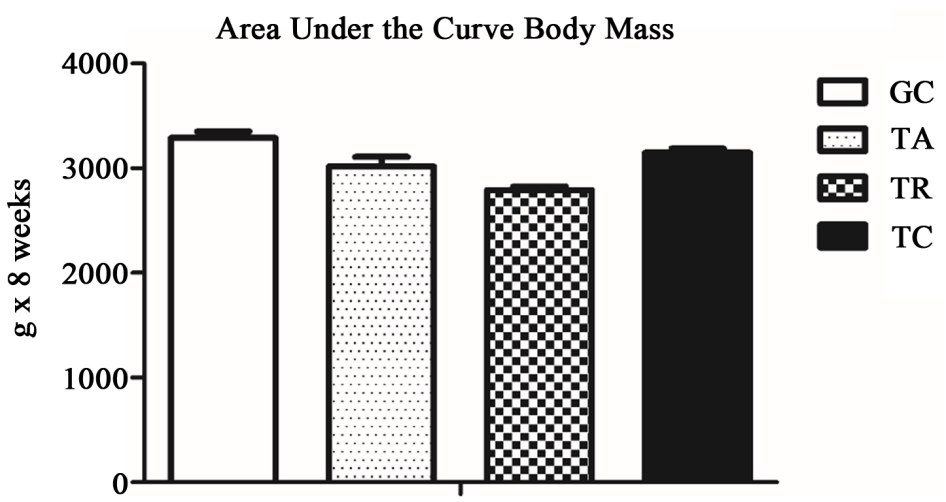

(b)

Figure 1. (a) Representative graph of the body weight evolution of the groups during the eight weeks of experiment; (b) Area under the curve of the body weight evolution of the groups during the eight weeks of experiment CG = Control Group, AT = Aerobic Training, $\mathrm{RT}=$ Resisted Training and $\mathrm{CT}=$ Concurrent Training. Values expressed as mean and standard error. ${ }^{*}$ Significant difference in relation to CG. 
TBARs

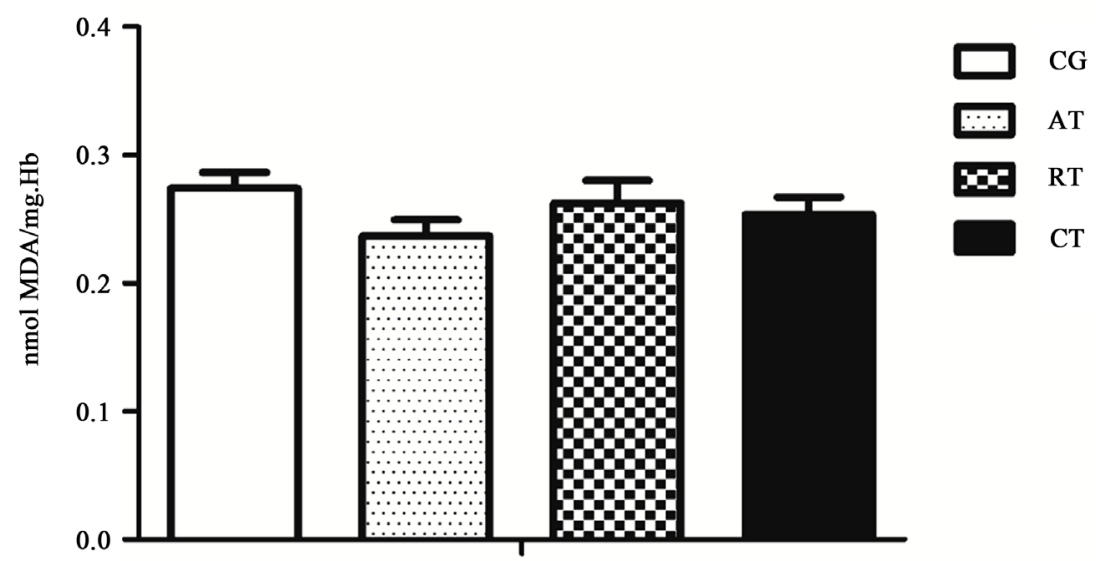

Figure 2. Concentration of thiobarbituric acid (TBARs) in erythrocytes in the control group (CG), aerobic training (AT), resistive training (RT) and concurrent training (CT) at the end of the experiment. Values expressed by mean and standard error. ${ }^{\star}$ Significant difference in relation to $\mathrm{CG}$.

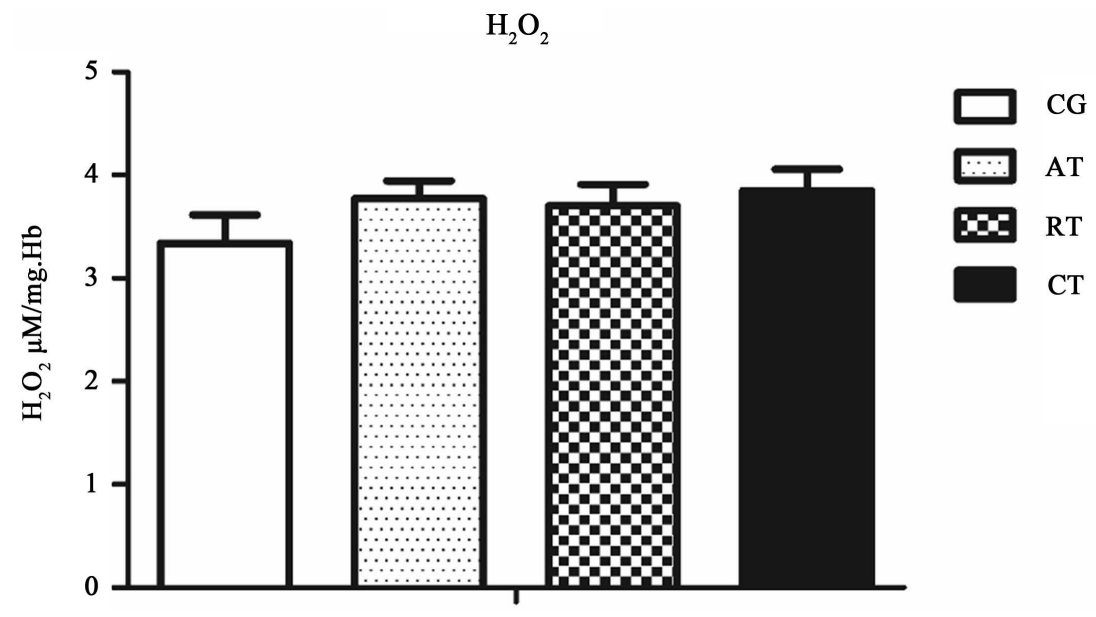

Figure 3. Concentration of hydrogen peroxide $\left(\mathrm{H}_{2} \mathrm{O}_{2}\right)$ in erythrocytes in the control group (CG), aerobic training (AT), resistive training (RT) and concurrent training (CT) at the end of the experiment. Values expressed by mean and standard error. ${ }^{\star}$ Significant difference in relation to CG.

To evaluate the antioxidant capacity the levels of the following enzymes were measured: catalase (CAT), glutathione peroxidase (GPx) and superoxide dismutase (SOD). Figure 4(a) shows the concentration values of CAT. In this figure we can observe that the three physical training protocols did not change the levels of CAT in erythrocytes in Wistar rats. By contrast, in Figure 4(b) (concentrations of GPx) and Figure 4(c) (concentrations of SOD) all the trainings were effective in increasing concentrations of these enzymes.

In order to evaluate the redox state in erythrocytes, we measured the ratio between the amount of reduced glutathione (GSH) and oxidized glutathione (GSSG). In this evaluation, it was not found significant difference between groups (Figure 5). 


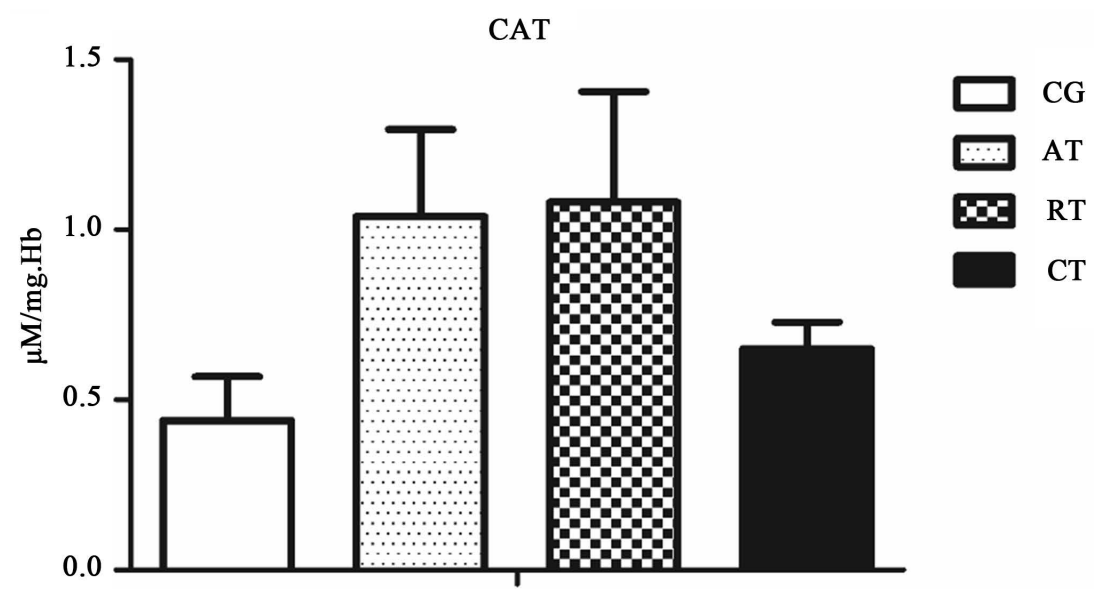

(a)

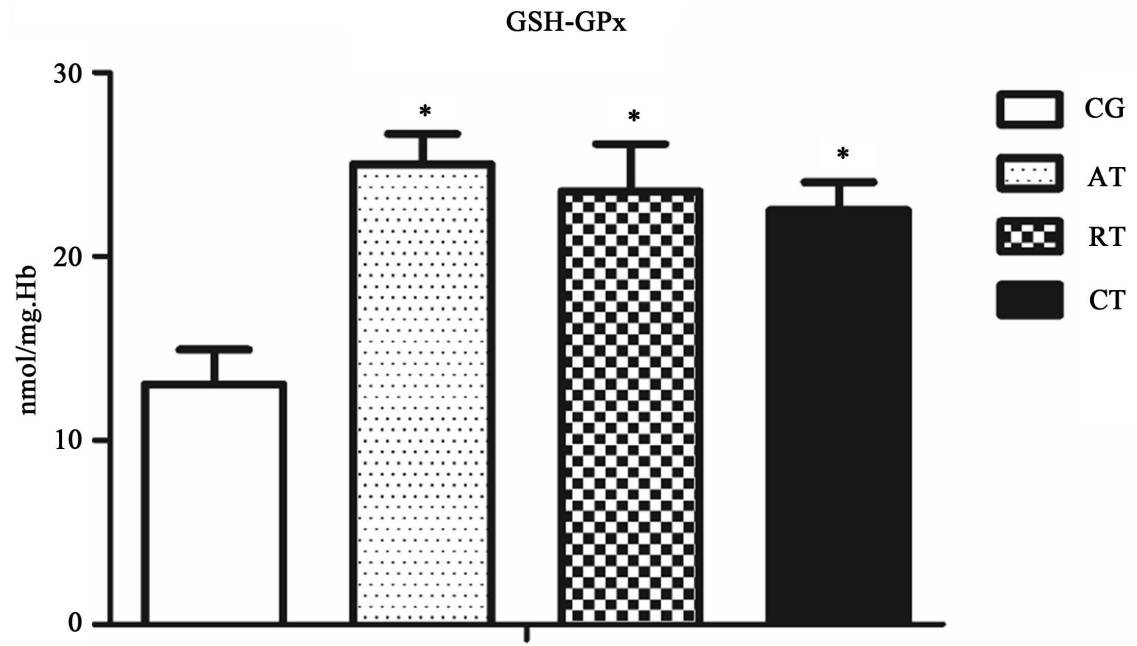

(b)

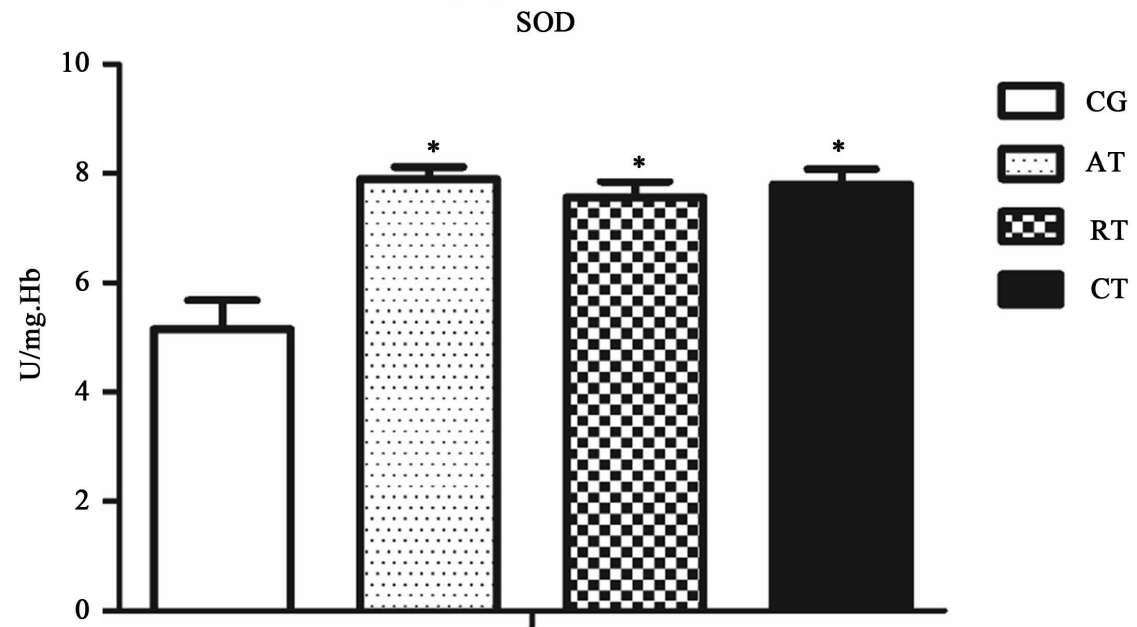

(c)

Figure 4. (a) Activity of enzymes Catalase (CAT); (b) GlutathionePeroxidase (GSH-GPx); (c) Superoxide Dismutase in erythrocytes in the control group (CG), aerobic training (AT), resistive training (RT) and concurrent training (CT) at the end of the experiment. Values expressed by mean and standard error. ${ }^{\star}$ Significant difference in relation to CG. 


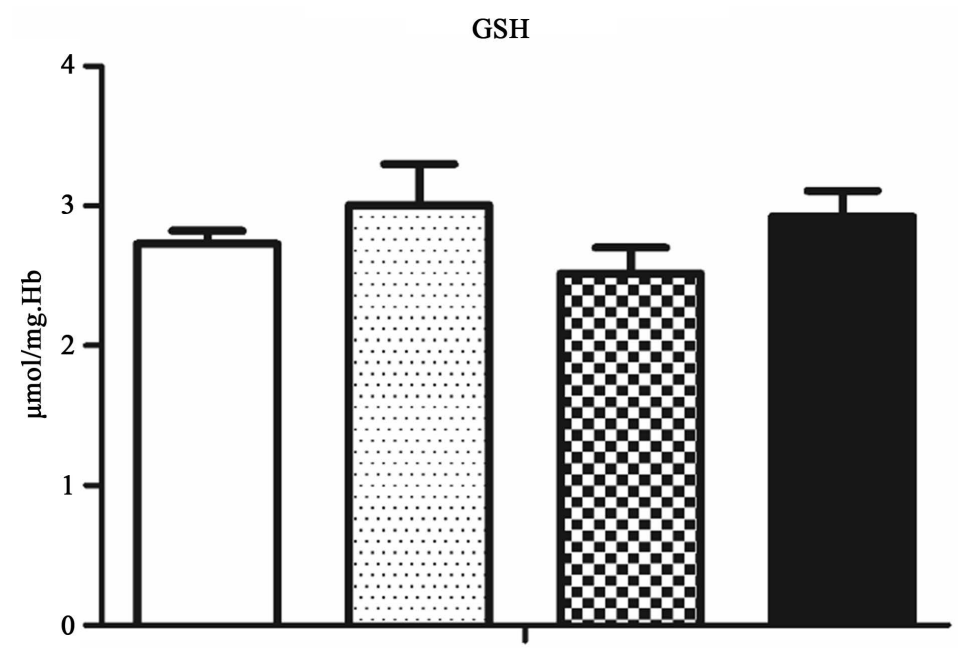

(a)

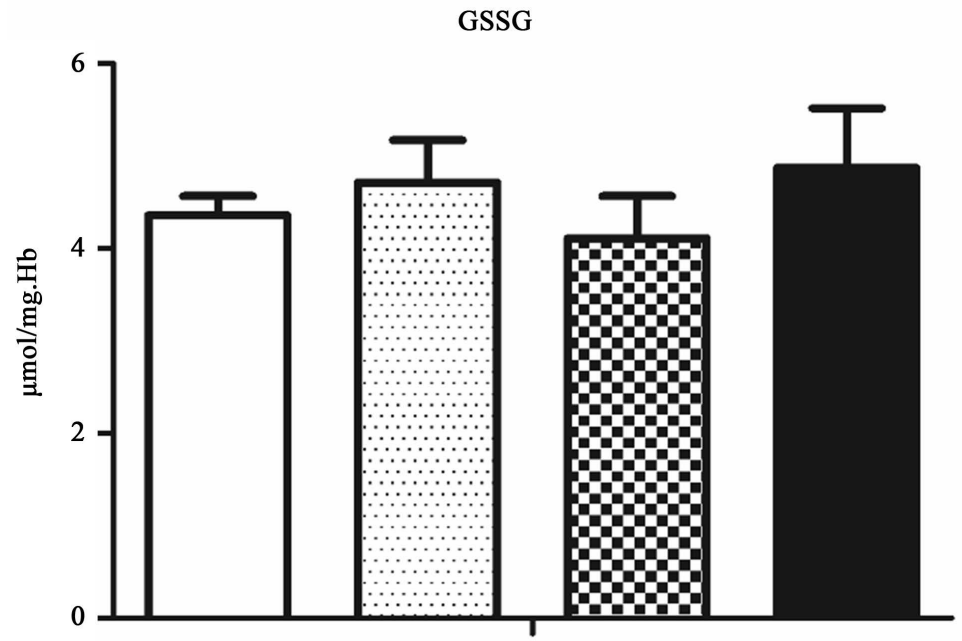

(b)

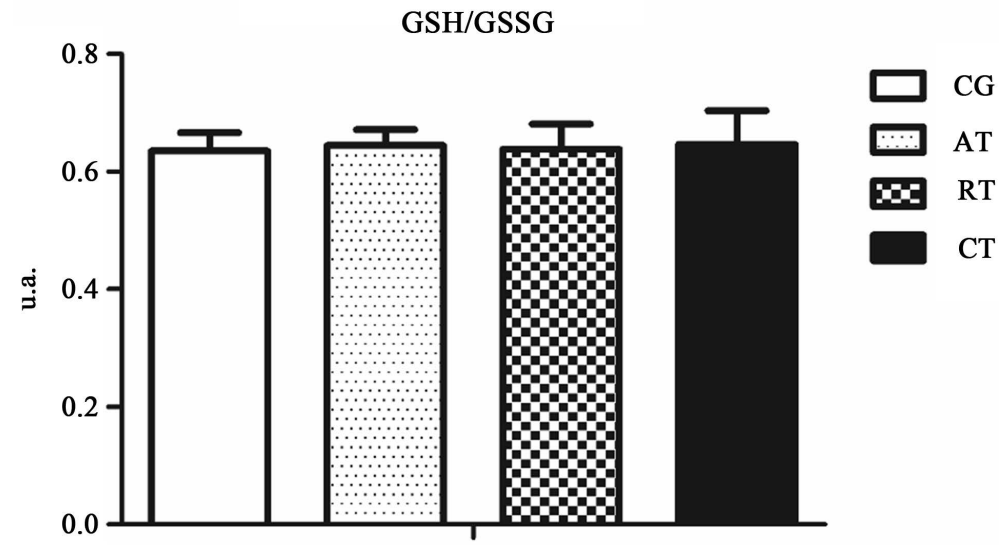

(c)

Figure 5. Activity of the enzymes reduced glutathione (GSH), oxidized glutathione (GSSG) and reduced/oxidized ratio (GSH/GSSG) in erythrocytes in the control group (CG), aerobic training (AT), resistive training (RT) and concurrent training (CT) at the end of the experiment. Values expressed by mean and standard error. ${ }^{*}$ Significant difference in relation to $C G$. 


\section{Discussion}

Since the time when the researchers have found that a session of physical exercise promotes an increase in reactive oxygen species (ROS), several studies aiming to investigate the effect of exercise training on these variables have been made. There are some studies showing that exercise training can promote a decrease in state of oxidative stress, i.e., may reduce levels of lipid peroxidation and ROS, but also increase the antioxidant system [7] [35]. These benefits can occur either by aerobic training [18] [19] [20] [36] as by the resistive training [18] [21] [22] [23]. A combination of stimuli (aerobic and resistive) in animal models had not been studied, so the differential of this study was to investigate the effect of three different protocols (aerobic, resistive and concurrent) on markers of oxidant systems $\left(\mathrm{H}_{2} \mathrm{O}_{2}\right.$ concentrations and TBARs) and antioxidant (CAT, SOD, GSH-GPx and GSH/GSSG).

In the present study we analyzed the concentrations of lipid peroxidation by the method of analysis of substances formation which react to thiobarbituric acid (TBARs). TBARs is a molecule formed in the early lipid peroxidation of polyunsaturated fatty acids when they are attacked by radicalar and not radicalar species. Although the TBARs measurement test is very unspecific, it can provide an idea about the process of lipid peroxidation [37]. In our study, the different training protocols did not cause changes in lipid peroxidation in erythrocytes in Wistar rats under resting conditions.

Moreover, studies according to Sertuk et al. [12] andOztsan et al. [20] using protocols of aerobic training on a treadmill observed no changes in the levels of lipid peroxidation in erythrocytes of rats in the resting condition. Therefore training protocols used in these studies have demonstrated efficacy in not allowing an increase in lipid peroxidation marker after exhaustive exercise. A limitation of our study is that we did not perform the evaluations after acute exercise. Possibly training protocols could lead to cellular adaptation which would promote a lower lipid peroxidation after acute bout of exercise. Corroborating our findings, Araújo et al. (2010) have found effects of different training protocols (25\% above and below the maximal lactate steady state) in the levels of lipid peroxidation in erythrocytes and have verified that physical training protocols, either aerobic or anaerobic, do not promote changes in the levels of marker of lipid peroxidation.

Another evaluation performed in order to measure the levels of pro-oxidant molecules was hydrogen peroxide $\left(\mathrm{H}_{2} \mathrm{O}_{2}\right)$. It is known that high levels of ROS promote harmful effects on cells compromising its functions [2] [3]. In contrast, physiological levels of these substances are necessary for distinct cellular signaling pathways [4] [5] [6] [7]. No physical training protocol promoted changes in this variable.

A lower ROS production is not enough to get a protected cellular redox state, but it is also necessary that the antioxidant system would be with its full functionally to protect cells from damaging effects caused by ROS [38]. For this rea- 
son, to examine the effect of different protocols in the antioxidant capacity, we analyzed the activity of the enzymes superoxide dismutase (SOD), glutathione peroxidase (GSH-GPx), catalase (CAT) and the ratio between reduced glutathione (GSH) and oxidized glutathione (GSSG).

Regarding the antioxidant enzymes SOD and GSH-GPx, it was found significant increases for all physical training protocols studied, although no differences were found in CAT concentrations in animals of trained groups compared to the sedentary group. The SOD enzyme has the function of dismutate the superoxide radical (O2•-) in hydrogen peroxide $\left(\mathrm{H}_{2} \mathrm{O}_{2}\right)$, which can subsequently be catalyzed by enzymes CAT and GSH-GPx, being converted into water and molecular oxygen. The results of this study are in agreement with some studies in the literature, showing that training can increase the activity of SOD in erythrocytes [39] and other tissues [40] [41].

Increased levels of GSH-Px caused by the three physical training protocols are consistent with some studies. According to Oztasan et al. [20] and Miyazaki et al. [39] aerobic physical training promotes significant increases in the levels of GSH-GPx in erythrocytes in rats. This effect caused by training in the activity of SOD and GSH-GPx, probably occurs in response to oxidative stress induced by several consecutive sessions of exercise. According to Tiidus et al. [42] and Gomez-Cabrera et al. [43] regular physical training, whether aerobic or resistive, stimulates the increase in concentrations of protein kinase 5' adenosine monophosphate (AMPK) cellular, which is responsible for triggering the activation of nuclear factor kappa B (NF-kB). This transcription factor NF-kB is responsible for triggering a variety of genes, including SOD and GSH-Px, resulting in a possible adaptation arising of training protocols used in this study.

The effect of training on antioxidant enzymes, mainly catalase activity and expression is still inconsistent and controversial. Pinho et al. [44] have found that a 12-week training increases levels of SOD and reduces levels of CAT, thus proposing that an overload could occur in the formation of $\mathrm{H}_{2} \mathrm{O}_{2}$ possibly causing harmful effects on cells due to the ease of peroxide to react with transition metals and generate hydroxyl radical which is the most reactive existing radial [45]. These authors have not reported the existence of a redox imbalance, since levels of the activity GSH-GPx and $\mathrm{H}_{2} \mathrm{O}_{2}$ were not measured in this study.

However, in our study, we measured the activity of GSH-GPx and found significant increases in all trained groups (aerobic, resistive and concurrent). The GSH-GPx and CAT possess similar functions with regard to the decomposition of $\mathrm{H}_{2} \mathrm{O}_{2}$. In relation to this aspect, the GSH-GPx is more activated in the presence of high concentrations of $\mathrm{H}_{2} \mathrm{O}_{2}$, whereas CAT performs an important function in the presence of low concentrations of $\mathrm{H}_{2} \mathrm{O}_{2}$ [46]. One hypothesis for the results aforementioned is that physical training performed five times per week with a high amount (in the three protocols) provided an adaptive effect on the redox balance of antioxidants, making the high concentrations of $\mathrm{H}_{2} \mathrm{O}_{2}$ to suffer primarily action from GSH-GPx. 
Other variables measured in our study were the concentrations of GSH, GSSG and GSH/GSSG ratio, which were not modified by any of the physical training protocols. The ratio between GSH and GSSG represents the cellular redox state, since the GSH plays a multifunctional role in protecting tissues against oxidative damage during exercise assisting in the direct removal of $\mathrm{H}_{2} \mathrm{O}_{2}$ being transformed into GSSG, and is also used in preventing lipid peroxidation [47]. There are studies that have demonstrated that exercise training can promote an increase in GSH content and reduce GSSG muscular [47] [48]. In contrast, studies have reported a reduction in GSH content in rat soleus muscle after training [49] or increase in GSH and GSSG enzymes, leading to maintenance of GSH/GSSG ratio in erythrocytes [50].

Furthermore, is worth emphasizing that the GSH synthesis in skeletal muscle occurs at low rates, thus promoting an import of GSH by muscle coming from the plasma and/or erythrocytes. Muscle tissue not only is capable of importing GSH, but also exporting GSSG. Thus, if the GSH consumption rate exceeds the production rate, a maintenance may occur or even a decrease in the GSH/GSSG ratio post-training in erythrocytes, so that skeletal muscle may combat internal oxidative stress [47]. This phenomenon may explain the increase in certain antioxidant variables and the maintenance of GSH/GSSG ratio in erythrocytes in all training protocols used in our study.

The initial hypothesis of the study was that concurrent training should induce an adaptation of the antioxidant system that would overcome the aerobic and resistive protocols separately, depending on the stimulation of production of reactive oxygen species. But this study showed a similar adaptation in the three training protocols used. Possibly the adaptations of the antioxidant system are induced by the amount of training and not specifically by stimulation of each of the training protocols. According to this hypothesis and with the results aforementioned Tromm et al. [51] have verified the effect of two and three days per week of aerobic training, and have observed that only the protocol of three days promoted adaptations in the antioxidant system and the protocol of two days was not enough to promote the benefits resulting from physical training.

\section{Conclusion}

Therefore, we can conclude that the three physical training protocols promote an increase in antioxidant enzymes without alteration of pro-oxidant markers and modifications in redox balance.

\section{References}

[1] Dillard, C.J., Litov, R.E., Savin, W.M., Dumelin, E.E. and Tappel, A.L. (1978) Effects of Exercise, Vitamin E, and Ozone on Pulmonary Function and Lipid Peroxidation. Journal of Applied Physiology, 45, 927-932. https://doi.org/10.1152/jappl.1978.45.6.927

[2] Forbes, J.M., Coughlan, M.T. and Cooper, M.E. (2008) Oxidative Stress as a Major Culprit in Kidney Disease in Diabetes. Diabetes, 57, 1446-1454. 
https://doi.org/10.2337/db08-0057

[3] Briones, A.M. and Touyz, R.M. (2010) Oxidative Stress and Hypertension: Current Concepts. Current Hypertension Reports, 12, 135-142.

https://doi.org/10.1007/s11906-010-0100-Z

[4] Burke, T.M. and Wolin, M.S. (1987) Hydrogen Peroxide Elicits Pulmonary Arterial Relaxation and Guanylate Cyclase Activation. American Journal of Physiology, 252, H721-H732. https://doi.org/10.1152/ajpheart.1987.252.4.H721

[5] Galter, D., Mihm, S. and Droge, W. (1994) Distinct Effects of Glutathione Disulphide on the Nuclear Transcription Factor Kappa B and the Activator Protein-1. European Journal of Biochemistry, 221, 639-648. https://doi.org/10.1111/j.1432-1033.1994.tb18776.x

[6] Powers, S.K., Talbert, E.E. and Adhihetty, P.J. (2011) Reactive Oxygen and Nitrogen Species as Intracellular Signals in Skeletal Muscle. The Journal of Physiology, 589, 2129-2138. https://doi.org/10.1113/jphysiol.2010.201327

[7] Villanueva, C. and Kross, R.D. (2012) Antioxidant-Induced Stress. International Journal of Molecular Sciences, 13, 2091-2109. https://doi.org/10.3390/ijms13022091

[8] Davies, K.J., Quintanilha, A.T., Brooks, G.A. and Packer, L. (1982) Free Radicals and Tissue Damage Produced by Exercise. Biochemical and Biophysical Research Communications, 107, 1198-1205. https://doi.org/10.1016/S0006-291X(82)80124-1

[9] Powers, S.K. and Jackson, M.J. (2008) Exercise-Induced Oxidative Stress: Cellular Mechanisms and Impact on Muscle Force Production. Physiological Reviews, 88, 1243-1276. https://doi.org/10.1152/physrev.00031.2007

[10] Veskoukis, A.S., Nikolaidis, M.G., Kyparos, A. and Kouretas, D. (2009) Blood Reflects Tissue Oxidative Stress Depending on Biomarker and Tissue Studied. Free Radical Biology and Medicine, 47, 1371-1374. https://doi.org/10.1016/j.freeradbiomed.2009.07.014

[11] Nikolaidis, M.G., Kyparos, A. and Vrabas, I.S. (2012) Cell Redox Homeostasis: Reading Conti et al. Data from a Blood-Centric Perspective. Medicine \& Science in Sports \& Exercise, 44, 190, Author Reply 191. https://doi.org/10.1249/MSS.0b013e318230e29f

[12] Senturk, U.K., Yalcin, O., Gunduz, F., Kuru, O., Meiselman, H.J. and Baskurt, O.K. (2005) Effect of Antioxidant Vitamin Treatment on the Time Course of Hematological and Hemorheological Alterations after an Exhausting Exercise Episode in Human Subjects. Journal of Applied Physiology, 98, 1272-1279. https://doi.org/10.1152/japplphysiol.00875.2004

[13] Veskoukis, A.S., Nikolaidis, M.G., Kyparos, A., Kokkinos, D., Nepka, C., Barbanis, S. and Kouretas, D. (2008) Effects of Xanthine Oxidase Inhibition on Oxidative Stress and Swimming Performance in Rats. Applied Physiology Nutrition and $\mathrm{Me}$ tabolism, 33, 1140-1154. https://doi.org/10.1139/H08-102

[14] Nikolaidis, M.G., Kyparos, A. and Vrabas, I.S. (2011) F(2)-Isoprostane Formation, Measurement and Interpretation: The Role of Exercise. Progress in Lipid Research, 50, 89-103. https://doi.org/10.1016/j.plipres.2010.10.002

[15] Conti, V., Corbi, G., Russomanno, G., Simeon, V., Ferrara, N., Filippelli, W., Limongelli, F., Canonico, R., Grasso, C., Stiuso, P., Dicitore, A. and Filippelli, A. (2012) Oxidative Stress Effects on Endothelial Cells Treated with Different Athletes' Sera. Medicine \& Science in Sports \& Exercise, 44, 39-49. https://doi.org/10.1249/MSS.0b013e318227f69c

[16] Radak, Z., Pucsuk, J., Boros, S., Josfai, L. and Taylor, A.W. (2000) Changes in Urine 8-Hydroxydeoxyguanosine Levels of Super-Marathon Runners during a Four-Day 
Race Period. Life Sciences, 66, 1763-1767. https://doi.org/10.1016/S0024-3205(00)00499-9

[17] Fisher-Wellman, K. and Bloomer, R.J. (2009) Acute Exercise and Oxidative Stress: A 30 Year History. Dynamic Medicine, 8, 1. https://doi.org/10.1186/1476-5918-8-1

[18] da Silva, L.A., Pinho, C.A., Rocha, L.G., Tuon, T., Silveira, P.C. and Pinho, R.A. (2009) Effect of Different Models of Physical Exercise on Oxidative Stress Markers in Mouse Liver. Applied Physiology, Nutrition, and Metabolism, 34, 60-65. https://doi.org/10.1139/H08-132

[19] Revan, S. and Erol, A.E. (2011) Effects of Endurance Training on Exhaustive Exercise-Induced Oxidative Stress Markers. African Journal of Pharmacy and Pharmacology, 5, 437-441. https://doi.org/10.5897/AJPP11.159

[20] Oztasan, N., Taysi, S., Gumustekin, K., Altinkaynak, K., Aktas, O., Timur, H., Siktar, E., Keles, S., Akar, S., Akcay, F., Dane, S. and Gul, M. (2004) Endurance Training Attenuates Exercise-Induced Oxidative Stress in Erythrocytes in Rat. European Journal of Applied Physiology, 91, 622-627. https://doi.org/10.1007/s00421-003-1029-6

[21] Parise, G., Brose, A.N. and Tarnopolsky, M.A. (2005) Resistance Exercise Training Decreases Oxidative Damage to DNA and Increases Cytochrome Oxidase Activity in Older Adults. Experimental Gerontology, 40, 173-180. https://doi.org/10.1016/j.exger.2004.09.002

[22] Vincent, H.K., Bourguignon, C. and Vincent, K.R. (2006) Resistance Training Lowers Exercise-Induced Oxidative Stress and Homocysteine Levels in Overweight and Obese Older Adults. Obesity (Silver Spring), 14, 1921-1930. https://doi.org/10.1038/oby.2006.224

[23] Murlasits, Z., Cutlip, R.G., Geronilla, K.B., Rao, K.M., Wonderlin, W.F. and Alway, S.E. (2006) Resistance Training Increases Heat Shock Protein Levels in Skeletal Muscle of Young and Old Rats. Experimental Gerontology, 41, 398-406. https://doi.org/10.1016/j.exger.2006.01.005

[24] Ahmadiasl, N., Najafipour, H., Soufi, F.G. and Jafari, A. (2012) Effect of Short- and Long-Term Strength Exercise on Cardiac Oxidative Stress and Performance in Rat. Journal of Physiology and Biochemistry, 68, 121-128. https://doi.org/10.1007/s13105-011-0125-Z

[25] Tegtbur, U., Busse, M.W. and Braumann, K.M. (1993) Estimation of an Individual Equilibrium between Lactate Production and Catabolism during Exercise. Medicine \& Science in Sports \& Exercise, 25, 620-627. https://doi.org/10.1249/00005768-199305000-00015

[26] Jones, A.M. and Doust, J.H. (1998) The Validity of the Lactate Minimum Test for Determination of the Maximal Lactate Steady State. Medicine \& Science in Sports \& Exercise, 30, 1304-1313. https://doi.org/10.1097/00005768-199808000-00020

[27] de Araujo, G.G., Papoti, M., Manchado Fde, B., de Mello, M.A. and Gobatto, C.A. (2007) Protocols for Hyperlactatemia Induction in the Lactate Minimum Test Adapted to Swimming Rats. Comparative Biochemistry and Physiology Part A: Molecular \& Integrative Physiology, 148, 888-892.

https://doi.org/10.1016/j.cbpa.2007.09.002

[28] de Araujo, G.G., Papoti, M., Dos Reis, I.G., de Mello, M.A. and Gobatto, C.A. (2012) Physiological Responses during Linear Periodized Training in Rats. European Journal of Applied Physiology, 112, 839-852. https://doi.org/10.1007/s00421-011-2020-2

[29] Voltarelli, F.A., Gobatto, C.A. and de Mello, M.A. (2002) Determination of Anae- 
robic Threshold in Rats using the Lactate Minimum Test. Brazilian Journal of Medical and Biological Research, 35, 1389-1394. https://doi.org/10.1590/S0100-879X2002001100018

[30] Engel, P.C. and Jones, J.B. (1978) Causes and Elimination of Erratic Blanks in Enzymatic Metabolite Assays Involving the Use of NAD+ in Alkaline Hydrazine Buffers: Improved Conditions for the Assay of L-Glutamate, L-Lactate, and Other Metabolites. Analytical Biochemistry, 88, 475-484.

https://doi.org/10.1016/0003-2697(78)90447-5

[31] Mujika, I., Chaouachi, A. and Chamari, K. (2010) Precompetition Taper and Nutritional Strategies: Special Reference to Training During Ramadan Intermittent Fast. British Journal of Sports Medicine, 44, 495-501.

[32] Rogatto, G.P., Oliveira, Cam., Faria, M.C. and Luciano, E. (2004) Respostas metabólicas agudas de ratos Wistar ao exercício intermitente de saltos. Motriz, Rio Claro, 10, 61-66.

[33] Kuipers, H. (1998) Training and Overtraining: An Introduction. Medicine \& Science in Sports \& Exercise, 30, 1137-1139. https://doi.org/10.1097/00005768-199807000-00018

[34] Ohkawa, H., Ohishi, N. and Yagi, K. (1979) Assay for Lipid Peroxides in Animal Tissues by Thiobarbituric Acid Reaction. Analytical Biochemistry, 95, 351-358. https://doi.org/10.1016/0003-2697(79)90738-3

[35] Powers, S.K., Nelson, W.B. and Hudson, M.B. (2011) Exercise-Induced Oxidative Stress in Humans: Cause and Consequences. Free Radical Biology \& Medicine, 51, 942-950. https://doi.org/10.1016/j.freeradbiomed.2010.12.009

[36] Qi, Z., He, J., Zhang, Y., Shao, Y. and Ding, S. (2011) Exercise Training Attenuates Oxidative Stress and Decreases p53 Protein Content in Skeletal Muscle of Type 2 Diabetic Goto-Kakizaki Rats. Free Radical Biology \& Medicine, 50, 794-800. https://doi.org/10.1016/j.freeradbiomed.2010.12.022

[37] Janero, D.R. (1990) Malondialdehyde and Thiobarbituric Acid-Reactivity as Diagnostic Indices of Lipid Peroxidation and Peroxidative Tissue Injury. Free Radical Biology \& Medicine, 9, 515-540. https://doi.org/10.1016/0891-5849(90)90131-2

[38] Lambertucci, R.H., Levada-Pires, A.C., Rossoni, L.V., Curi, R. and Pithon-Curi, T.C. (2007) Effects of Aerobic Exercise Training on Antioxidant Enzyme Activities and mRNA Levels in Soleus Muscle from Young and Aged Rats. Mechanisms of Ageing and Development, 128, 267-275. https://doi.org/10.1016/j.mad.2006.12.006

[39] Miyazaki, H., Oh-ishi, S., Ookawara, T., Kizaki, T., Toshinai, K., Ha, S., Haga, S., Ji, L.L. and Ohno, H. (2001) Strenuous Endurance Training in Humans Reduces Oxidative Stress Following Exhausting Exercise. European Journal of Applied Physiology, 84, 1-6. https://doi.org/10.1007/s004210000342

[40] Navarro-Arevalo, A. and Sanchez-del-Pino, M.J. (1998) Age and Exercise-Related Changes in Lipid Peroxidation and Superoxide Dismutase Activity in Liver and Soleus Muscle Tissues of Rats. Mechanisms of Ageing and Development, 104, 91-102. https://doi.org/10.1016/S0047-6374(98)00061-X

[41] Hamilton, K.L., Powers, S.K., Sugiura, T., Kim, S., Lennon, S., Tumer, N. and Mehta, J.L. (2001) Short-Term Exercise Training Can Improve Myocardial Tolerance to I/R without Elevation in Heat Shock Proteins. American Journal of Physiology-Heart and Circulatory Physiology, 281, H1346-H1352. https://doi.org/10.1152/ajpheart.2001.281.3.H1346

[42] Tiidus, P.M. and Houston, M.E. (1994) Antioxidant and Oxidative Enzyme Adaptations to Vitamin E Deprivation and Training. Medicine \& Science in Sports \& Exer- 
cise, 26, 354-359. https://doi.org/10.1249/00005768-199403000-00012

[43] Gomez-Cabrera, M.C., Domenech, E. and Vina, J. (2008) Moderate Exercise Is an Antioxidant: Upregulation of Antioxidant Genes by Training. Free Radical Biology \& Medicine, 44, 126-131. https://doi.org/10.1016/j.freeradbiomed.2007.02.001

[44] Pinho, R.A., Andrades, M.E., Oliveira, M.R., Pirola, A.C., Zago, M.S., Silveira, P.C., Dal-Pizzol, F. and Moreira, J.C. (2006) Imbalance in SOD/CAT Activities in Rat Skeletal Muscles Submitted to Treadmill Training Exercise. Cell Biology International, 30, 848-853. https://doi.org/10.1016/j.cellbi.2006.03.011

[45] Halliwell, B. and Gutteridge, J.M.C. (2007) Free Radicals in Biology and Medicine. Clarendon Press, Oxford.

[46] Jenkins, R.R. and Goldfarb, A. (1993) Introduction: Oxidant Stress, Aging, and Exercise. Medicine \& Science in Sports \& Exercise, 25, 210-212. https://doi.org/10.1249/00005768-199302000-00008

[47] Ji, L.L. (1995) Oxidative Stress during Exercise: Implication of Antioxidant Nutrients. Free Radical Biology \& Medicine, 18, 1079-1086.

https://doi.org/10.1016/0891-5849(94)00212-3

[48] Ferreira, J.C., Bacurau, A.V., Bueno, C.R., Jr., Cunha, T.C., Tanaka, L.Y., Jardim, M.A., Ramires, P.R. and Brum, P.C. (2010) Aerobic Exercise Training Improves $\mathrm{Ca}^{2+}$ Handling and Redox Status of Skeletal Muscle in Mice. Experimental Biology and Medicine (Maywood), 235, 497-505. https://doi.org/10.1258/ebm.2009.009165

[49] Leeuwenburgh, C., Fiebig, R., Chandwaney, R. and Ji, L.L. (1994) Aging and Exercise Training in Skeletal Muscle: Responses of Glutathione and Antioxidant Enzyme Systems. American Journal of Physiology, 267, R439-R445.

[50] Janiak, M., Suska, M., Dudzinska, W. and Skotnicka, E. (2010) Blood Glutathione Status and Activity of Glutathione-Metabolizing Antioxidant Enzymes in Erythrocytes of Young Trotters in Basic Training. Journal of Animal Physiology and Animal Nutrition (BerI), 94, 137-145. https://doi.org/10.1111/j.1439-0396.2008.00889.x

[51] fluorometric method, C.B., Rosa, G.L.D., Bom, K., Mariano, I., Pozzi, B., Tuon, T., Silva, L.A. and Pinho, R.A. (2012) Effect of Different Frequencies Weekly Training on Parameters of Oxidative Stress. Brazilian Journal Kinanthropometry and Human Performance, 14, 52-60. 